\title{
Revelación de Información de Responsabilidad Social Empresaria en el Sector Financiero Argentino. Factores Explicativos
}

\author{
Disclosure Determinants of Corporate Social \\ Responsibility Information in the Argentinian \\ Financial Sector
}

\author{
Sandra Patricia Pastor ${ }^{1}$, Pablo Raffaelli² ${ }^{2}$ Mónica Cristina Jugón ${ }^{3}$
}

\section{RESUMEN}

En el presente trabajo, se analiza la cantidad de información divulgada sobre la gestión social, económica y ambiental de las entidades financieras argentinas y los factores explicativos de la revelación.

En base a los fundamentos de la teoría de los costos políticos se estudian los determinantes de la divulgación voluntaria de información corporativa a través de un modelo de regresión lineal.

Los resultados obtenidos concuerdan con estudios anteriores (Gamerschlag et al., 2011), asociándose de manera positiva la cantidad de información de Responsabilidad Social Empresaria (RSE) divulgada con una mayor visibilidad de la empresa, una estructura de propiedad de los accionistas más dispersa y la auditoria de los informes de RSE, resultando inversa su relación con la rentabilidad.

Las conclusiones arribadas se fortalecen a partir de la desagregación del índice general en los aspectos ambiental, social y económico de manera particular.

Palabras clave: divulgación de información, responsabilidad social empresaria, sector financiero.

Recepción: 24/05/2019, Aprobación: 16/08/2019

\footnotetext{
1 Departamento de Contabilidad, Auditoria y Costos, Facultad de Ciencias Económicas, Universidad Nacional del Centro de la Provincia de Buenos Aires, Sede Tandil, Argentina. Correo: sandra.pastor@econ.unicen.edu.ar

2 Departamento de Contabilidad, Auditoria y Costos, Facultad de Ciencias Económicas, Universidad Nacional del Centro de la Provincia de Buenos Aires, Sede Tandil, Argentina. Correo: pablo.raffaelli@econ.unicen.edu.ar

3 Departamento de Administración, Facultad de Ciencias Económicas, Universidad Nacional del Centro de la Provincia de Buenos Aires, Sede Tandil, Argentina. Correo: monica.jugon@econ.unicen.edu.ar
} 


\begin{abstract}
In this paper, the amount of information disclosed on the social, economic and environmental management of argentine financial institutions and the explanatory factors of the disclosure is analysed.

Based on the fundamentals of the theory of political costs, the determinants of the voluntary disclosure of corporate information are studied through a linear regression model.

The results obtained agree with previous studies (Gamerschlag et al., 2011), associating positively the amount of CSR information disclosed with greater visibility of the company, a structure of ownership of the most dispersed shareholders and the audit of reports of CSR, its relationship with profitability being inverse.

The conclusions obtained are strengthened from the disaggregation of the general index in the environmental, social and economic aspects in a particular way.
\end{abstract}

Keywords: corporate social responsibility, information disclosure, financial sector.

\title{
INTRODUCCIÓN
}

La información de Responsabilidad Social (RS) que divulgan las empresas, depende de la decisión estratégica de la gerencia o la administración por tratarse de un acto voluntario.

Las diversas teorías que estudian la divulgación de información corporativa asumen que, en determinadas circunstancias, las empresas divulgan más información cuando las expectativas de la relación costo-beneficio resultan favorables, sin embargo, existen particularidades de cada organización que influyen en la estrategia de revelación.

A partir del análisis de la cantidad de información revelada en relación a los determinantes de la divulgación más significativos, podrá observarse el perfil de las organizaciones que emisoras de información de RS. Con este objetivo, se trabaja con un índice de revelación global (IRG) que se desagrega en los tres aspectos que involucran a la RS: índice de revelación económica (IRE), índice de revelación ambiental (IRA) e índice de revelación social (IRS).

Se seleccionaron entidades financieras pertenecientes a la Asociación de Bancos de Argentina, que presentan de manera voluntaria Reportes de Sustentabilidad bajo el formato de la Guía del Global Reporting Initiative (GRI), con la finalidad de trabajar con una base de datos homogénea en términos de contexto macroeconómico y social. Se consideraron cuatro periodos de reporte entre los años 2014 y 2017, lo que resultó en un valor final de 30 observaciones empresa-año. Se excluye el factor sector de actividad como determinante de la revelación por resultar común a toda la población, a pesar de que la literatura lo considera relevante.

Numerosos estudios empíricos tratan de explicar, desde una perspectiva económica, la existencia de una vinculación entre la decisión de revelación y las características de las empresas que las llevan adelante, (Cooke, 1992; Giner, 1997; Archambault y Archambault, 2003).

Según Blacconiere y Northcut (1997), los grupos relacionados con la empresa por motivos 
financieros o económicos encuentran de utilidad la información social y ambiental revelada en el proceso de toma de decisiones.

El diseño del índice de medición de la información divulgada es una tarea compleja afectada por la subjetividad, en el que incide la selección de elementos y la ponderación (García-Meca y Martínez, 2004). Su trascendencia radica en la capacidad de captar los valores de las variables independientes cuya significancia se intenta explicar (Cho et al, 2015). Por lo tanto el índice construido para medir cantidad de información social y ambiental divulgada, debe garantizar una adecuada capacidad de representación del objeto analizado desde el enfoque teórico previsto.

Diversas metodologías de trabajo han sido observadas. Reverte (2009) utilizó la regresión lineal múltiple en empresas cotizadas de España para analizar la relación entre la RSE y cada uno de los factores que influyen en su divulgación, como el tamaño de la empresa, la sensibilidad de la industria, la rentabilidad, la concentración en la estructura de capital, cotización internacional, exposición a los medios y apalancamiento. La técnica de análisis estadístico también fue empleada por Gamerschlag et al. (2011) para identificar los determinantes de la divulgación voluntaria de la RSE en las empresas alemanas, utilizando como factores la visibilidad de la empresa, la rentabilidad, la estructura de los accionistas y la relación con las partes interesadas.

La información de responsabilidad social es la destinada a revelar el impacto ambiental y social generado por la organización, teniendo en cuenta los diversos grupos involucrados (Gray et al., 2001), cuya revelación cobra trascendencia a partir de las limitaciones de la información financiera (Larrinaga et al., 2002).

La política de RS de la empresa integra el valor corporativo revelado voluntariamente, con independencia de cualquier marco regulatorio que así lo indique, quedando la decisión de revelación a cargo de los gerentes o administradores, excepto por aspectos medioambientales y del ámbito de los RRHH que deben informarse en cumplimiento de normativas vigentes.

A partir de las diversas teorías en las que se sustenta el estudio de la divulgación de información voluntaria; teoría de la agencia, teoría de la señal, teoría de los costos de información, teoría de la legitimación y teoría de los costos políticos entre otras, se trata de relacionar la cantidad de información revelada con determinados perfiles corporativos.

Basados principalmente en la teoría de la señal, el grado de visibilidad o exposición en los medios de comunicación por parte de las empresas, afecta las expectativas del valor actual neto de la firma y por ende su valor bursátil.

A su vez, las ventajas y desventajas de la divulgación pueden fundamentarse a partir de la teoría de los costos políticos. Las partes interesadas presionan a la gerencia según su nivel de poder, legitimidad y urgencia del reclamo interpuesto. Los factores que explican la divulgación en base a la teoría de los costos políticos son: visibilidad de la empresa, rentabilidad, la estructura del capital, la relación con accionistas de otros mercados (EEUU).

En esta línea, Gamerschlag et al. (2011), analizan algunas variables independientes en un estudio sobre empresas alemanas. Sus hallazgos permiten vincular la visibilidad, la estructura de 
capital, y la participación en mercados de capitales externos (EEUU), con la diversidad y cantidad de información de RSE divulgada.

Los determinantes de la divulgación seleccionados para el presente trabajo, pueden ser explicados a la vez desde los fundamentos de otras teorías, fundamentalmente la teoría de la legitimidad y stakeholders, por cuanto no resultan excluyentes.

La teoría de la legimitidad reconoce que las empresas están limitadas por un contrato social donde asumen compromisos para recibir un beneficio económico a cambio o bien garantizar su continuidad (Brown y Deegan 1998). Supone que las organizaciones hacen propios los valores que la sociedad como colectivo fomenta, y por ende premia a quienes los impulsan. Esta teoría es particularmente tenida en cuenta cuando ocurren situaciones que cuestionan las decisiones del ente quedando expuesto al juicio de la opinión pública (Deegan et al., 2002).

La teoría de los stakeholders reconoce las responsabilidades de la empresa con todas las partes interesadas (Garriga y Melé, 2004), que afectan directamente a la organización, con un alcance más restringido que en el caso de la legitimidad que considera la sociedad como un conjunto.

La teoría de la agencia ve a la empresa como un contrato entre varios agentes con intereses en conflicto que actúan en un mercado considerado eficiente (Reverte, 2008). Gray et al. (1995) expusieron que los gerentes usan la información de la compañía para satisfacer o manipular a las partes interesadas influyentes y asi obtener el apoyo que se requiere para la supervivencia. De allí la coincidencia con el carácter político del análisis que propone.

\section{Hipótesis de la investigación}

H1 La visibilidad de las empresas está asociada positivamente con la divulgación de la RSE. Algunas compañías se encuentran más expuestas a la opinión pública, por lo que la mayor divulgación de información social y ambiental, permite reducir los posibles costos políticos de sus decisiones (Belkaoui y Karpic, 1989).

H2 La dispersión en la propiedad del capital se asocia con una mayor divulgación de información de RSE. Cuando existe alta concentración en la propiedad del capital, la presión es menor y la gerencia se encontrará menos dispuesta a brindar información en caso que sea requerida. Por el contrario, cuando la propiedad se dispersa la necesidad de consensuar apoyos y evitar asimetrías de información, trae como resultado una mayor divulgación.

H3 La oferta pública en la Bolsa de Nueva York posee asociación positiva con la divulgación de información sobre responsabilidad social. Dado que en EEUU existe una alta discrecionalidad de las empresas respecto a la estrategia de divulgación, las organizaciones asumen la responsabilidad de decidir respecto a qué y cuanta información revelar. Por esta razón las empresas extrajeras que se financian en el mercado de capitales norteamericano asumen modalidades de divulgación habituales para las empresas cotizantes (Matten y Moon, 2008).

H4 La rentabilidad se asocia de manera positiva con la divulgación de RSE. Las empresas con mayores utilidades están obligadas a intensificar las explicaciones sobre el origen del superávit en tanto 
existan otras competidoras menos rentables con buena reputación y cuentas con recursos para afrontar costos de divulgación que en caso contrario podría quedar fuera de presupuesto (Brammer y Pavelin, 2006). Consideraremos la Rentabilidad Financiera (ROE) y la Rentabilidad Económica (ROA) esta última respecto a la divulgación de indicadores económicos.

H5 Existe una relación positiva entre la divulgación de RSE y la auditoria de los Reportes de Sustentabilidad. El cumplimiento de procedimientos de aseguramiento de la información voluntaria presentada actúa como una limitante a la discrecionalidad de la gerencia respecto a la decisión de divulgación. La incorporación de esta hipótesis es un aporte de esta investigación, en el marco de la teoría de los costos políticos.

Se incorpora como hipótesis adicional respecto a la divulgación de información económica el endeudamiento (END), considerando que el mayor valor se asocia a mayor divulgación.

Como variable de control se utiliza la variable tamaño (TAM), considerando como tal el Activo Total de cada empresa ponderado por el logaritmo natural.

A continuación, se describe el diseño y la metodología de trabajo, para continuar con la presentación de resultados y conclusiones. Se finaliza con la identificación de oportunidades para profundizar la línea de trabajo.

\section{METODOLOGÍA, MATERIALES Y MÉTODOS}

Para generar una base de datos homogénea, se optó por considerar empresas bajo un mismo contexto político, social y económico seleccionando las entidades financieras pertenecientes a la Asociación de Bancos de Argentina., que hayan reportado sus Informes de RSE cumpliendo con lo enmarcado en los lineamientos del GRI.

Se consideraron cuatro periodos de reporte entre los años 2014 y 2017, lo que resultó en un valor final de 30 observaciones empresa-año.

\section{Variables Dependientes}

Se procederá a analizar el comportamiento de los factores explicativos de la divulgación (Cuadro 1), en relación con la cantidad de información revelada, a partir de un índice de revelación general y su desagregación en torno a los aspectos sociales, ambientales y económicos de la RSE, identificados como variables dependientes del estudio.

- Indice de revelación general: es un índice de cantidad de información divulgada, que se calcula como la relación entre el número de indicadores realmente informados en sus reportes anuales por parte de las empresas consideradas en el estudio, con el número total de indicadores posibles a informar en cada una de las tres áreas de la RSE (económico, ambiental y social).

- Índice de revelación económico: relaciona cantidad de indicadores económicos efectivamente divulgados con los indicadores económicos propuestos por el GRI.

- Índice de revelación ambiental: relaciona cantidad de indicadores ambientales efectivamente divulgados con los indicadores ambientales propuestos por el GRI. 
- Índice de revelación social: relaciona cantidad de indicadores sociales divulgados con los indicadores sociales propuestos por el GRI.

\section{Cuadro 1}

\section{Variables dependientes}

\begin{tabular}{|c|ccc|}
\hline \multirow{2}{*}{$I R G=\frac{i E D+i A D+i S D}{i E T+i A T+i S T}$} & $I R E=\frac{i E D}{i E T}$ & $I R A=\frac{i A D}{i A T}$ & IRS $=\frac{i S D}{i S T}$ \\
\hline \multirow{3}{*}{$\begin{array}{c}\text { IRG = Índice } \\
\text { Revelación General }\end{array}$} & $\begin{array}{c}\text { IRE }=\text { Índice } \\
\text { Revelación Económico }\end{array}$ & $\begin{array}{c}\text { IRA }=\text { Índice } \\
\text { Revelación Ambiental }\end{array}$ & $\begin{array}{c}\text { IRS }=\text { Índice } \\
\text { Revelación Social }\end{array}$ \\
\cline { 2 - 4 } & $\begin{array}{c}\mathrm{iED}=\text { Indicadores } \\
\text { Económicos Divulgados }\end{array}$ & $\begin{array}{c}\text { iAD }=\text { Indicadores } \\
\text { Ambientales Divulgados }\end{array}$ & $\begin{array}{c}\text { *iSD }=\text { Indicadores } \\
\text { Sociales Divulgados }\end{array}$ \\
\cline { 2 - 4 } & iET = Indicadores & iAT $=$ Indicadores & iST = Indicadores \\
& Económicos Totales & Ambientales Totales & Sociales Totales \\
\hline
\end{tabular}

Fuente: Elaboración propia. Índices utilizados en el análisis de los factores explicativos de la divulgación sobre RSE.

\section{Variables Independientes}

Como variables independientes se plantean los determinantes de divulgación de la RSE y una variable de control (tamaño).

\section{Determinantes de la RSE.}

- Visibilidad de la empresa (VISIB): está íntimamente relacionada con la atención mediática que recibe una compañía. Se midió contando el número de noticias sobre la empresa que aparecían en cada año ante la búsqueda exacta de su nombre en el portal web del diario de negocios "Ámbito Financiero", vocero de la comunidad empresaria con eje en las finanzas. En adición, para validar la medición de esta variable, se repitió este procedimiento en dos páginas de diarios electrónicos con mayor tráfico web en los últimos años, como lo son "IProfesional" y "El Cronista", reflejando valores muy similares a las del caso tomado, dándole validez al mismo.

- Concentración del capital (FREEFLOAT): esta información fue tomada de los Estados Financieros Anuales de las empresas para los cuatro ejercicios, como el porcentual libre del accionista mayoritario en cada compañía. Cuanto más alto mayor es la dispersión.

- Cotización en el mercado cambiario de Estados Unidos (COTUSA): se estableció la variable dicotómica de si las empresas cotizaban en la Bolsa de Comercio de Nueva York. Tomando valor " 1 " si lo hacían, y "0" en caso contrario.

- Rentabilidad (ROE): para el cálculo de esta variable se utiliza la formula (Beneficio antes de impuestos / Patrimonio promedio) multiplicado por cien (datos obtenidos de los Estados Financieros Anuales), dando como resultado el ratio de rentabilidad financiera.

- Auditoria de RSE (AUDRSE): se presenta como una variable dicotómica, que tomará valor “1” 
si los Reportes de Sustentabilidad fueron auditados, y "0" en caso contrario.

- Para analizar la divulgación de información económica se utilizan variables propias del análisis económico financiero, complementadas con la visibilidad de la empresa como variable cualitativa más influyente de acuerdo a trabajos previos en la temática. Los factores explicativos que se adicionan son: Rentabilidad económica (ROA) calculada como el cociente entre Ganancia Actividades Operativas y el Activo total; el Endeudamiento (END) que relaciona el Pasivo total con el Patrimonio al cierre.

- Variable de control: Tamaño de la firma (TAM): se utilizó el monto de los Activos extraído de cada uno de los Estados Financieros Anuales de las compañías, como una medida comparativa del tamaño de las empresas. Cabe destacar que se utilizó el logaritmo natural para deflactar sus valores.

\section{Modelo de regresión}

Se utilizará un modelo de regresión lineal múltiple para analizar con técnicas cuantitativas la relación entre la cantidad de RSE informada y los factores que influyen en esa divulgación (Branco y Rodrigues, 2008; Gamerschlag, et al., 2011; Reverte, 2009).

Basándose en el uso del programa estadístico STATA para examinar la relación entre los factores que influyen en las cantidades divulgadas de RSE, tal como lo proponen las siguientes regresiones multivariadas (Cuadro 2):

\section{Cuadro 2}

\section{Modelos de regresión}

\begin{tabular}{|l|}
\hline$I R G=\beta_{0}+\beta_{1}$ VISIB $+\beta_{2}$ FREEFLOAT $+\beta_{3}$ COTUSA $+\beta_{4} R O E+\beta_{5}$ AUDRSE $+\beta_{6}$ TAM $+\varepsilon$ \\
\hline$I R A=\beta_{0}+\beta_{1}$ VISIB $+\beta_{2}$ FREEFLOAT $+\beta_{3}$ COTUSA $+\beta_{4} R O E+\beta_{5}$ AUDRSE $+\beta_{6} T A M+\varepsilon$ \\
\hline$I R S=\beta_{0}+\beta_{1}$ VISIB $+\beta_{2}$ FREEFLOAT $+\beta_{3}$ COTUSA $+\beta_{4} R O E+\beta_{5} A U D R S E+\beta_{6} T A M+\varepsilon$ \\
\hline$I R E=\beta_{0}+\beta_{1}$ VISIB $+\beta_{2}$ ROE $+\beta_{3} E N D+\beta_{4} R O A+\beta_{5} T A M+\varepsilon$ \\
\hline
\end{tabular}

Nota: Elaboración propia donde, IRG (Índice Revelación General); VISIB (Visibilidad de la empresa); FREEFLOAT (distribución de la estructura de capital); COTUSA (Cotización en la Bolsa de Comercio de Nueva York); ROE (Rentabilidad Financiera); AUDRSE (Auditoria de los Reportes de Sustentabilidad); TAM (Tamaño de la firma); IRA (Índice Revelación Ambiental); IRS (Índice Revelación Social); IRE (Índice Revelación Económico); END (Endeudamiento); ROA (Rentabilidad Económica).

\section{RESULTADOS}

\section{Estadísticas Descriptivas y Correlaciones}

La Tabla 1 presenta las estadísticas descriptivas para todas las variables de interés incluidas en el modelo. Reflejándose un total de 30 observaciones, y siendo la media de la variable dependiente (porcentaje de indicadores divulgados sobre el total a informar) IRG de 42,12, tomando valores 
que parten desde 21,98 hasta 72,53. En cuanto a las variables independientes, se presentan dos de ellas como dicotómicas, COTUSA y AUDRSE con medias de 0,767 y 0,467 respectivamente. Las variables independientes restantes presentan las siguientes medias: TAM $(17,75), \operatorname{ROE}(45,96)$, VISIB $(188,40)$ y FREEFLOAT $(28,18)$.

Cuando se trabaja con muestras de menos de 100 observaciones es necesario verificar si los errores cumplen una distribución normal. Esta se ha verificado mediante el análisis de Skewness y Kurtosis, con valores aproximadamente normales que se vieron reducidos debido a la utilización del logaritmo natural de los activos en la variable Tamaño (TAM).

\section{Tabla 1}

\section{Estadísticas descriptivas y normalidad de los errores}

\begin{tabular}{|lccccccc|}
\hline Variable & N & Min. & Max. & Media & Desv. Std. & Kurtosis & Skewness \\
\hline IRG & 30 & 21,98 & 72,53 & 42,12 & 15,07 & 2,034 & 0,434 \\
\hline TAM & 30 & 13,21 & 19,63 & 17,75 & 1,660 & 5,642 & $-1,801$ \\
\hline ROE & 30 & 18,40 & 74,20 & 45,96 & 14,29 & 2,629 & $-0,480$ \\
\hline COTUSA & 30 & 0 & 1 & 0,767 & 0,430 & 2,590 & $-1,261$ \\
\hline AUDRSE & 30 & 0 & 1 & 0,467 & 0,507 & 1,018 & 0,134 \\
\hline VISIB & 30 & 1 & 554 & 188,4 & 194,4 & 1,793 & 0,688 \\
\hline FREEFLOAT & 30 & 0,0100 & 80,29 & 28,18 & 26,60 & 2,343 & 0,607 \\
\hline
\end{tabular}

Fuente: Elaboración propia. Estadísticas descriptivas y normalidad de los errores.

La Tabla 2 muestra las correlaciones de Pearson para el índice de divulgación, las variables independientes y la variable de control. Las correlaciones entre las variables independientes no proporcionan indicios de multicolinealidad, ya que ninguna correlación bivariada excede el valor de 0,9 (Hair et al. 2010; Peng y Beamish 2008). Se observa que la cantidad de revelación de RSE está correlacionada positivamente en un alto grado y con un nivel de significancia del $1 \%$ con la Auditoria de los Reportes de Sustentabilidad (AUDRSE) en 0,4774 y con la Visibilidad de la empresa (VISIB) en un 0,4056. En adición, proporciona evidencia de que la variable dependiente está correlacionada estadísticamente de manera positiva con la dispersión de los capitales (FREEFLOAT) en un 0,1149 (nivel de significancia del 5\%), y de manera negativa con un nivel de significancia del 10\% con la rentabilidad (ROE) por un valor de - 0,2574. 
Tabla 2

Correlación de Pearson

\begin{tabular}{|lccccccc|}
\hline \multicolumn{1}{|c|}{ IRG } & TAM & ROE & COTUSA & AUDRSE & VISIB & FREEFLOAT \\
\hline IRG & 1,0000 & & & & & & \\
\hline TAM & $-0,1241$ & 1,0000 & & & & & \\
\hline ROE & $-0,2574^{* * *}$ & 0,4824 & 1,0000 & & & & \\
\hline COTUSA & 0,1598 & 0,7012 & 0,0824 & 1,0000 & & & \\
\hline AUDRSE & $0,4774^{*}$ & $-0,1965$ & 0,0710 & 0,0421 & 1,0000 & & \\
\hline VISIB & $0,4056^{*}$ & 0,4690 & 0,2641 & 0,4885 & 0,3293 & 1,0000 & \\
\hline FREEFLOAT & $0,1149^{* *}$ & 0,4785 & 0,1711 & 0,5943 & 0,4055 & 0,6181 & 1,0000 \\
\hline
\end{tabular}

Nota: *Significancia a nivel 0,01. ** Significancia a nivel 0,05. ** Significancia a nivel 0,10, y elaboración propia.

Se puede observar que la variable independiente dicotómica de cotización en la Bolsa de Nueva York (COTUSA) no presenta correlación con la cantidad de información de RSE divulgada, como tampoco la variable de control tamaño (TAM).

\section{Análisis de Regresión}

Si bien los análisis descriptivos y de correlación brindan información sobre el nivel promedio y las relaciones univariadas entre las variables, sus efectos conjuntos son aún más preponderantes. Se aplicó el análisis de regresión para estimar estos efectos, utilizando los índices de revelación global y desagregados, como las variables dependientes.

\section{Variable dependiente: Índice Revelación General (IRG)}

La Tabla 3 permite contrastar las hipótesis planteadas. La H1 se verifica la asociación positiva de la visibilidad de las empresas con la divulgación de RSE ( $p$ value $=0,01$ ); la H2, confirma la relación positiva entre la dispersión del capital (mayor freefloat) y la variable dependiente ( $\mathrm{p}$ value $=0,05)$; la H3 no verifica asociación entre cotización en USA y divulgación; se verifica relación entre la rentabilidad financiera y la variable dependiente (H4), aunque en proporción inversa a la estimada debido a que poseen una relación negativa y a un nivel de significancia menor ( $p$ value = 0,10); la relación entre la divulgación de RSE y el cumplimiento de auditoria (H5) es altamente significativa y de valor positivo ( $p$ value $=0,05$ ).

El modelo en su conjunto posee un coeficiente de determinación alto en relación con estudios de este tipo (Reverte, 2009; Gamerschlag et al., 2011; Branco y Rodrigues, 2008), lo que da por cumplida la calidad del modelo para replicar los resultados. (Prob $>F=0,004$; R-cuadrado=0,532; $\mathrm{R}$-cuadrado Ajustado=0,409). En consecuencia, se concluye que tanto la visibilidad de la compañía, la dispersión de su capital y la auditoria de la RSE, son determinantes importantes en la mayor divulgación de indicadores de responsabilidad social bajo formato del GRI que se incluyen en los Reportes de Sustentabilidad de las entidades financieras de Argentina. 
Tabla 3

Análisis de Regresión - Determinantes de divulgación de RSE

\begin{tabular}{|c|c|c|c|c|c|}
\hline IRG & Coef. & St. Err & t-value & p-value & Sig. \\
\hline TAM & $-0,087$ & 2,694 & $-0,03$ & 0,975 & \\
\hline $\mathrm{ROE}$ & $-0,386$ & 0,201 & $-1,92$ & 0,068 & $* * *$ \\
\hline COTUSA & 6,738 & 8,609 & 0,78 & 0,442 & \\
\hline AUDRSE & 15,063 & 5,803 & 2,60 & 0,016 & ** \\
\hline VISIB & 0,042 & 0,015 & 2,74 & 0,012 & * \\
\hline FREEFLOAT & $-0,266$ & 0,126 & $-2,11$ & 0,046 & $* *$ \\
\hline _cons & 48,874 & 38,879 & 1,26 & 0,221 & \\
\hline R-squared & \multicolumn{2}{|c|}{0,532} & \multicolumn{2}{|c|}{ Num of obs } & 30,000 \\
\hline Adjusted R-squared & \multicolumn{2}{|c|}{0,409} & \multicolumn{2}{|c|}{ Prob $>$ F } & 0,004 \\
\hline F-test & \multicolumn{2}{|c|}{4,351} & \multicolumn{2}{|c|}{ Akaike crit } & 238,120 \\
\hline
\end{tabular}

Nota: * Significancia a nivel 0,01. ** Significancia a nivel $0,05 .{ }^{* *}$ Significancia a nivel 0,10 y elaboración propia.

Los hallazgos alcanzados son en su mayoría consistentes con trabajos anteriores sobre revelaciones ambientales en otros países (Chen y Bouvain, 2009; Bayoud et al., 2012; Brammer y Pavelin, 2006; Gamerschlag et al., 2011). Además, los resultados obtenidos son en gran medida coherentes con las hipótesis derivadas de la teoría del costo político, lo que sugiere que las empresas divulgan información para reducir la regulación y los impuestos potenciales (Watts y Zimmermann 1978).

El análisis propuesto se complementa con la desagregación del Índice de Revelación General en cada una de las dimensiones específicas de la responsabilidad social, y su relación con los determinantes explicativos de la revelación de información seleccionados para el estudio.

En la Tabla 4 se presenta la verificación de las hipótesis planteadas considerando como variable dependiente el Índice de Revelación Ambiental.

\section{Tabla 4}

Variable dependiente: Índice Revelación Ambiental (IRA).

\begin{tabular}{|lccccc|}
\hline & Visibilidad & Freefloat & Auditoría RSE & ROE & Cotización USA \\
\hline Significativo al 0.01 & + & & & & \\
\hline Significativo al 0.10 & & & + & & \\
\hline No significativo & & $\mathrm{x}$ & & $\mathrm{x}$ & $\mathrm{x}$ \\
\hline
\end{tabular}

Fuente: Elaboración propia. Determinantes de divulgación en base al Índice de Revelación Ambiental. 
Los resultados obtenidos muestran un coeficiente de determinación alto, en relación a estudios precedentes, lo que da por cumplida la calidad del mismo para replicar los resultados. (Prob $>$ F=0,0087; R-cuadrado=0,50; R-cuadrado Ajustado=0,37).

Se observa que tanto la visibilidad de la compañía y la auditoria de la RSE, son factores explicativos relevantes positivos de la mayor divulgación de indicadores medioambientales.

La Tabla 5 presentada a continuación, refleja la relación de las hipótesis planteadas considerando como variable dependiente el Índice de Revelación Social.

Tabla 5

Variable dependiente: Índice Revelación Social (IRS).

\begin{tabular}{|lccccc|}
\hline & Visibilidad & Freefloat & Auditoría RSE & ROE & Cotización USA \\
\hline Significativo al 0.05 & & + & + & - & \\
\hline No significativo & $\begin{array}{c}\mathrm{X} \\
\text { (p value 0,14) }\end{array}$ & & & & $\mathrm{x}$ \\
(p value 0,14)
\end{tabular}

Fuente: Elaboración propia. Determinantes de divulgación en base al Índice de Revelación Social.

La regresión lineal obtenida muestra un coeficiente de determinación alto, lo que da por cumplida la calidad del mismo para replicar los resultados. (Prob $>F=0,0076$; R-cuadrado=0,51; Rcuadrado Ajustado=0,38).

Se concluye que tanto la estructura del capital (FREEFLOAT) como la auditoría del reporte de sustentabilidad, son factores explicativos relevantes de la mayor divulgación de indicadores medioambientales; en tanto que la Rentabilidad Financiera se asocia de manera inversa aunque significativa con la variable dependiente. La visibilidad de la empresa y la cotización en el mercado de valores de Nueva York no resultan significativas por escaso margen cuando se considera la cantidad de información social revelada.

Completando la desagregación del Índice de Revelación General se contrastan las hipótesis planteadas con el Índice de Revelación Económico, cuyos resultados se exponen en la Tabla 6.

\section{Tabla 6}

Variable dependiente: Índice Revelación Económico (IRE).

\begin{tabular}{|lccccc|}
\hline & Visibilidad & ROE & Endeudamiento & ROA & $\begin{array}{c}\text { Tamaño } \\
\text { (Variable control) }\end{array}$ \\
\hline Significativo al 0.01 & & & & - & - \\
\hline Significativo al 0.1 & & & - & \\
\hline No significativo & $\mathrm{X}$ & $\mathrm{x}$ & & \\
\hline
\end{tabular}

Fuente: Elaboración propia. Determinantes de divulgación en base al Índice de Revelación Económico. 
Se ha explicado la variable dependiente IRE en función de determinantes de naturaleza económica y financiera, para enfocar el análisis de la divulgación de información económica en los reportes de sostenibilidad, a partir de la restricción de costo-beneficio, incorporando la teoría de los costos políticos a través de la visibilidad del ente.

La regresión obtenida muestra un coeficiente de determinación alto, lo que da por cumplida la calidad del mismo para replicar los resultados. (Prob $>\mathrm{F}=0,0042$; R-cuadrado=0,49; R-cuadrado Ajustado=0,39).

Se advierte que el endeudamiento y la rentabilidad económica se asocian inversamente y de manera significativa con la divulgación de indicadores económicos según GRI.

\section{DISCUSIÓN Y CONCLUSIONES}

Numerosos académicos han intentado durante tiempo determinar los motivos por los cuales las empresas divulgan voluntariamente información sobre aspectos ambientales y sociales. Nos basamos en la teoría de los costos políticos (Watts y Zimmermann 1978) para argumentar que las empresas informan sobre la RSE por razones económicas: intentan reducir sus costos políticos proporcionando información sobre su responsabilidad social (Fields et al. 2001).

Identificamos una serie de factores específicos de la empresa que pueden actuar como sustitutos de los costos políticos y, como tales, es probable que estén relacionados con la disposición de la empresa a divulgar voluntariamente la información de RSE.

Sobre la base de los indicadores específicos del marco del GRI, se procedió a la construcción de cuatro índices de cantidad de información de RSE divulgada para ocho entidades financieras argentinas durante el periodo 2014-2017. Nuestros resultados muestran que la divulgación de la RSE, medida por IRG, se asocia positivamente con una mayor visibilidad de la empresa, una estructura de propiedad de los accionistas más dispersa y un cumplimiento en la auditoria de los Reportes de Sustentabilidad presentados, valores concordantes con estudios anteriores como el de Gamerschlag et al. (2011). La rentabilidad se asocia de manera inversa a la esperada, lo que implica que las empresas con menor ROE incrementan la revelación de indicadores sobre RS para lograr un futuro aumento de sus márgenes.

La revelación de información ambiental, en base al IRA, presenta una asociación positiva y significativa con la visibilidad de las empresas, coincidentemente con aquellas auditan sus reportes de sustentabilidad.

Respecto a la revelación de información social, IRS, se observa una relación positiva y significativa con el FREEFLOAT y la Auditoría de la RSE, y una asociación inversa a la esperada con la Rentabilidad Financiera.

La divulgación de indicadores económicos se vincula de manera positiva con Rentabilidad Económica y el Tamaño de la entidad, y de forma inversa con Endeudamiento. 
A pesar de las limitaciones planteadas creemos que nuestros resultados proporcionan información importante sobre los determinantes de la divulgación de la RSE, respecto a un sector poco abordado en estudios sobre la temática.

Este trabajo presenta ciertas limitaciones al intentar estudiar un apartado dentro de las ciencias sociales, en este caso la divulgación de RSE, siendo de gran dificultad considerar todas las variables que influyen en él. Se advierte que el número empresas de la población objeto de estudio resulta escaso al considerarse solamente las que integran el sector financiero argentino. Resulta altamente recomendable realizar estudios en esta temática integrando todas las empresas argentinas, que presentan Reporte de Sostenibilidad, agregando un análisis por tipo de actividad o sector de la empresa, lo que enriquecerá las conclusiones de este e incrementará la cantidad de observaciones en el modelo.

El campo sigue abierto a futuras investigaciones, no obstante, el presente estudio contribuye a los trabajos previos, otorgando evidencia empírica y ayudando a comprender mejor los determinantes de la divulgación de RSE.

\section{REFERENCIAS BIBLIOGRÁFICAS}

Archambault, J.J.; Archambault, M.E. (2003) A multinational test of determinants of corporate disclosure. The International Journal of Accounting, 2003, vol. 38, issue 2, 173-194.

Bayoud, N.S.; Kavanagh, M.; Slaughter, G.(2012). CSR disclosure and corporate reputation in developing countries: case of Libya. Journal of Business and Policy Research, 7 (1). pp. 131-160.

Belkaoui, A.; Karpik, P. (1989) Determinants of the Corporate Decision to Disclose Social Information. Accounting, Auditing \& Accountability Journal, Vol. 2 Issue 1.

Blacconiere, W.G.; Northcut, W.D. (1997) Environmental Information and Market Reactions to Environmental Legislation. Journal of Accounting, Auditing and Finance, Vol 12, No. 2.

Brammer, S.; Pavelin, S. (2006) Voluntary environmental disclosures by large UK companies. J Bus Finan Account 33(7 and 8):1168-1188.

Branco, M. C.; Rodrigues, L. L. (2008). Factors influencing social responsibility disclosure by Portuguese companies. Journal of Business Ethics, 83, 685-701.

Brown, N.; Deegan, C. (1998) The public disclosure of environmental performance information-a dual test of media agenda setting theory and legitimacy theory, Accounting and Business Research, 29:1,21-41.

Cooke, T.E. (1992) The Impact of Size, Stock Market Listing and Industry Type on Disclosure in the Annual Reports of Japanese Listed Corporations, Accounting and Business Research, 22:87, 229-237.

Chen, S.; Bouvain, P. (2009) Is corporate responsibility converging? A comparison of corporate responsibility reporting in the USA, UK, Australia, and Germany. J Bus Ethics 87:299-317.

Cho, C.; Michelon, G.; Patten, D.; Roberts, R. (2015) CSR disclosure: the more things change...? Accounting, Auditing \& Accountability Journal, Vol. 28 Issue: 1, pp.14-35.

Deegan, C. (2002), "Introduction: The Legitimising Effect of Social and Environmental Disclosures - a Theoretical Foundation", Accounting, Auditing and Accountability Journal, Vol. 15 No. 3, pp. 282-311. 
Fields, T.D.; Lys, T.Z.; Vincent, L. (2001) Empirical research on accounting choice. Journal of Accounting and Economic 31:255-307.

García Meca, E., Martínez Conesa, I. (2004) Divulgación voluntaria de información empresaria. Índices de Revelación. Partida doble, pp. 66-77.

Garriga, E.; Melé, D. (2004) Corporate Social Responsibility Theories: Mapping the Territory. Journal of Business Ethics 53: 51.

Gamerschlag, R., Möller, K., \& Verbeeten, F. (2011). Determinants of voluntary CSR disclosure: empirical evidence from Germany. Review of Managerial Science, 5(2-3), 233-262.

Gray, R., Reza, K., Lavers, S. (1995). Corporate Social and Environmental Reporting - A Review of the Literature and a Longitudinal Study of UK Disclosure. Accounting, Auditing \& Accountability Journal. 8. 47-77.

Gray, R.; Javad, M.; Power, D., Sinclair, D. (2001). Social and Environmental Disclosure and Corporate Characteristics: A Research Note and Extension. Journal of Business Finance $\mathcal{E}$ Accounting. 28. 327-356.

Giner, B. (1997) The influence of company characteristics and accounting regulation on information disclosed by Spanish firms. European Accounting Review, vol. 6, issue 1,45-68.

Hair, J.F.; Black, W.C.; Babin, B.J.; Anderson, R.E. (2010) Multivariate data analysis. A global perspective. Pearson, Upper Saddle River.

Larrinaga, C.; Carrasco, F.; Correa, C.; Llena, F.; Moneva, J. (2002) Accountability and accounting regulation: the case of the Spanish environmental disclosure standard, European Accounting Review, 11:4, 723-740.

Matten, D.; Moon, J. (2008). "Implicit" and "explicit" CSR: A conceptual framework for a comparative understanding of CSR. The Academy of Management Review, 33.

Peng, G.Z.; Beamish, P.W. (2008) The effect of national corporate responsibility environment on Japanese foreign direct investment. Journal of Business Ethics 80:677-695.

Reverte, C. (2008) Determinants of CSR disclosures ratings by Spanish listed firms, Journal of Business Ethics, 88, 351-366.

Reverte, C. (2009). Determinants of corporate social responsibility disclosure ratings by Spanish listed firms. Journal of Business Ethics, 88(2), 351-366

Watts, R.L.; Zimmermann, J.L. (1978) Towards a positive theory of the determination of accounting standards. Accounting Review 53(1):112-134. 